\title{
Crystal Structure of Tetrabutylammonium Chloride Complex with Rac-1,1'-bi- 2-naphthol: The Inclusion of Surfactant Molecules by Hydrogen Bonded Binaphthol Molecules
}

\author{
Emmanuel MARFO-OWUSU ${ }^{* \dagger}$ and Amber L. THOMPSON** \\ *Department of Chemical Science, University of Energy and Natural Resources, Sunyani, Ghana \\ **Chemical Crystallography, Department of Chemistry, Chemistry Research Laboratory, University of Oxford, \\ Mansfield Road, Oxford OX1 3TA, UK
}

\begin{abstract}
The tetrabutylammonium chloride complex with rac-1,1'-bi-2-naphthol crystallizes in monoclinic space group $P 2{ }_{1} / n$, with unit-cell parameters of; $a=10.4941(1) \AA, b=26.3302(3) \AA, c=11.5290(1) \AA, \alpha=90^{\circ}, \beta=100.0154(5)^{\circ}, \gamma=90^{\circ}, V=$ 3137.05(5) $\AA^{3}$, and $Z=4$. The packing mode, hydrogen bonding network, and crystal data suggest that the crystal structure is isomorphous with the reported structure of a tetrabutylammonium bromide complex with rac-1,1'-bi-2-naphthol. ${ }^{2}$
\end{abstract}

(Received December 23, 2020; Accepted January 22, 2021; Published on web June 10, 2021)

Studies on molecular-recognition phenomenon in host-guest chemistry associated with non-covalent interactions generated by the effect of the conformation and shapes of molecules have received much attention due to its applications in crystal engineering and material science, such as knowing the material properties of organic solid- state systems. ${ }^{1} \quad$ The H-bond interactions, $\pi \cdots \pi, \mathrm{C}-\mathrm{H} \cdots \pi, \mathrm{C}-\mathrm{H} \cdots \mathrm{O}$ and $\mathrm{C}-\mathrm{H} \cdots$ halide interactions, are some of the primary forces that play significant role in molecular-recognition phenomenon in such supramolecular systems. Recently, our group reported on the effects of the

Table 1 Crystal data and experimental parameters of 1:1 TBAC/ BNP complex

Chemical formula: $\mathrm{C}_{36} \mathrm{H}_{50} \mathrm{ClNO}_{2}$

Formula weight $=564.25$

$T=150 \mathrm{~K}$

Crystal system: monoclinic Space group: $P 2{ }_{1} / n$

$a=10.4941(1) \AA$

$\alpha=90^{\circ}$

$b=26.3302(3) \AA$

$c=11.5290(1) \AA$

$V=3137.05(5) \AA^{3}$

$\beta=100.0154(5)^{\circ}$

$\gamma=90^{\circ}$

$Z=4$

$D_{\text {calc }}=1.19 \mathrm{~g} / \mathrm{cm}^{3}$

Radiation: Mo $K \alpha(\lambda=0.71073 \AA)$

$\mu($ Mo $K \alpha)=0.154 \mathrm{~mm}^{-1} \quad F(000)=1224$

Crystal size $=0.15 \times 0.18 \times 0.24 \mathrm{~mm}^{3}$

No. of reflections collected $=13843$

No. of independent reflections $=7136$

$\theta$ range for data collection: $5-27^{\circ}$

Data/Restraint $/$ Parameters $=5432 / 0 / 362$

Goodness-of-fit on $F^{2}=0.95$

$R$ indices $[I>2 \sigma(I)]: R_{1}=0.0453, w R_{2}=0.0925$

$R$ indices (all data) $R_{1}=0.0675, w R_{2}=0.1011$

$(\Delta / \sigma)_{\max }=0.001$

$(\Delta \rho)_{\max }=0.34 \mathrm{e}^{-3} \quad(\Delta \rho)_{\min }=-0.44 \mathrm{e}^{-3}$

Measurement: Nonius Kappa CCD diffractometer

Program system: CRYSTALS

Structure determination: SUPERFLIP

CCDC deposition number: 1007157

$\dagger$ To whom correspondence should be addressed.

E-mail: emmanuel_jp@yahoo.com molecular conformation and the chain length in tetra- $n$ alkylammonium halide complexes with non-planar aromatic molecule. ${ }^{2}$ Typically, studies on the crystal structures of tetrabutyl- and tetrapentylammonium bromide complexes with rac-1,1'-bi-2-naphthol (hereafter, TBAB/BNP and TPAB/BNP, respectively) have shown that the molecules of $\mathrm{TBAB} / \mathrm{BNP}$ are held in their aggregates by strong $\mathrm{H}$-bond $(\mathrm{C}-\mathrm{H}-\mathrm{Br})$ and weak $\mathrm{H}$-bonds; $\mathrm{C}-\mathrm{H} \cdot \cdots \mathrm{O}, \mathrm{C}-\mathrm{H} \cdots \mathrm{Br}$, and $\mathrm{C}-\mathrm{H} \cdots \pi$. The packing structures were found to be different from each other, as well as those in TBAB with planar aromatic molecules. The tetrabutylammonium cation exhibits conformational and orientational disorder in TBAB/BNP, while in TPAB/BNP the alkyl chains of the tetrapentylammonium cation adopt a biradial conformation in which there are two sets of paired chains. The tetrapentylammonium cations in TPAB/BNP entrap the BNP molecules in a manner that is attributed to the effect of an increase in the chain length as well as the influence of a biradial conformation in the tetrapentylammonium cation. However, in TBAB/BNP the naphthol planes form a hydrogen-bonded void, and entrap the tetrabutylammonium cation. The patterns of the H-bond network as well as the conformation of the tetra- $n$-alkylammonium cations contribute to the formation of voids that entrap either BNP or a tetra- $n$-alkylammonium cation. ${ }^{2}$

Per the knowledge revealed in this study, we were motivated to investigate TBAC and TPAC complexes with BNP; (hereafter, TBAC/BNP, TPAC/BNP) in order to understand the effect of changing the halide anions on the packing modes as well as the H-bond network, and to compare with those in TBAB/BNP and TPAB/BNP. After several attempts to obtain these complexes, we were successful to obtain only a TBAC/BNP complex.<smiles>Oc1ccc2ccccc2c1-c1c(O)ccc2ccccc12</smiles>

Fig. 1 Chemical structure of 1:1 TBAC/BNP. 
Thus, in this manuscript we report on the crystal structure of TBAC/BNP and compare its packing mode and molecular interactions with those in TBAB/BNP to enable us to understand whether changing the halide anion generates an isomorphous structure or not. The crystals were obtained within three days by the slow evaporation of a warmed acetone/ethylacetate $(20 \mathrm{~mL})$ mixture in which TBAC $(0.28 \mathrm{~g}, 1 \mathrm{mmol})$, and BNP $(0.29 \mathrm{~g}, 1 \mathrm{mmol})$ were dissolved. Crystal data, and data collection details are listed in Table 1. Data collection and cell refinement were carried out using DENZO-SMN. Structure solution was carried out by direct methods using the program SUPERFLIP within the CRYSTALS software suite, and refined by full-matrix least-squares methods based on $F^{2}$. All $\mathrm{H}$ atoms were located in the electron density difference maps. The $\mathrm{H}$ atoms were positioned with an idealized geometry with $\mathrm{C}-\mathrm{H}=$ $0.93-0.99 \AA$, and $\mathrm{O}-\mathrm{H}=0.82 \AA$, and refined isotropically using a riding model. The chemical structure is shown in Fig. 1, while the atom numbering follows the scheme indicated in Fig. 2. The asymmetric unit (Fig. 2) consists of a tetrabutylammonium cation, chloride anion $\left(\mathrm{Cl}^{-}\right)$, and BNP molecule. The TBAC/BNP crystallizes in monoclinic crystal system (space group $\left.P 2_{1} / n\right)$.

The rigid naphthol planes form a hydrogen-bonded void and entraps TBAC (Fig. 3) in a fashion similar to that observed in TBAB/BNP. ${ }^{2}$ Additionally in both complexes the packing mode, crystal data and hydrogen bonding network suggest that both structures are isomorphous. Thus, changing the halide $\left(\mathrm{X}^{-}\right) ; \mathrm{X}=\mathrm{Br}$ to $\mathrm{Cl}$ ) or counter anion of the tetrabutylammonium salt $\left.\left(\mathrm{C}_{4} \mathrm{H}_{9}\right)_{4} \mathrm{~N}^{+} \mathrm{X}^{-}\right)$complex does not affect the packing mode in both TBAB/BNP and TBAC/BNP. However, their packing mode is different from that in TPAB/BNP, as well as in TBAB complexes with phenol and catechol in which the phenol or catechol molecules are located in zigzag channels formed by the tetragonal-shaped tetrabutylammonium cation. The dihedral angle between the two naphthol planes, defined by $\mathrm{O} 2, \mathrm{C} 3$, $\mathrm{C} 4 \cdots, \mathrm{C} 8$ and $\mathrm{O} 11, \mathrm{C} 10, \mathrm{C} 12 \cdots, \mathrm{C} 9$ is $-87.3^{\circ}$ and are almost normal to each other as well as exhibit a cisoid $^{3}$ conformation with the $\mathrm{OH}$ groups adopting the cis mode. The bond lengths and angles in BNP are within the limit and normal. Unlike in $\mathrm{TPAB} / \mathrm{BNP},{ }^{2}$ the angles about the $\mathrm{N}-\mathrm{C}$ bond in the tetrabutylammonium cation in TBAC/BNP and TBAB/BNP do not vary significantly from the tetrahedral value $\left(109.5^{\circ}\right)$. Similarly, the bond lengths and angles in tetrabutylammonium cations are not significantly different from those reported on tetrabutylammonium bromide ${ }^{4}$ and its complexes with phenol, ${ }^{5}$ or with catechol. ${ }^{6}$ However, the torsion angle (C29-C30-C31C32 $\left.\left(-67.2^{\circ}\right)\right)$ in TBAC depicts a gauche bend conformation about $\mathrm{C} 30-\mathrm{C} 31$ in one of the alkyl chains. Thus, the tetrabutylammonium cation chain in TBAC/BNP also exhibits conformational and orientational disorder, which is evident in the packing structure (Fig. 3).

In TBAC/BNP, the structure is stabilized by $\mathrm{O}-\mathrm{H} \cdot . \mathrm{Cl}$

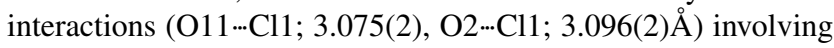
naphthol groups with the chloride anions to entrap the TBAC molecule in a similar $\mathrm{H}$-bond networks observed in TBAB/BNP. The stability of the structure is also ensured by interactions between a $\mathrm{H}$-atom of a methylene group of a tetrabutylammonium cation, and oxygen atom of hydroxyl moiety of BNP (H38... O11; $2.65 \AA$ and $\mathrm{C} 38 \ldots \mathrm{O} 11 ; 3.429(2) \AA, \mathrm{H} 262 \ldots \mathrm{O} 2 ; 2.54$ and $\mathrm{C} 2 \ldots \mathrm{O} 2$; $3.414(2) \AA$ ), as well as $\mathrm{C}-\mathrm{H} \cdot . \mathrm{Cl}$ contacts which exists between the $\mathrm{C}-\mathrm{H}$ of a naphthol moiety and chloride anion $(\mathrm{H} 41 \ldots \mathrm{Cl}$; 2.82, and $\mathrm{C} 4 \ldots \mathrm{Cl} 1 ; 3.570(2), \mathrm{H} 121 \ldots \mathrm{Cl} 1 ; 2.82 \AA$, and $\mathrm{C} 12 \cdots \mathrm{Cl} 1$; 3.074(2) $\mathrm{A}$, and also contacts between $\mathrm{C}-\mathrm{H}$ of an alkyl chain and

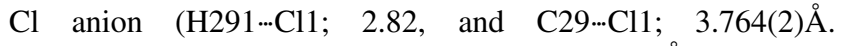

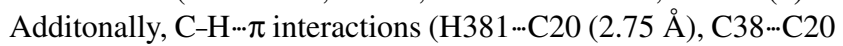

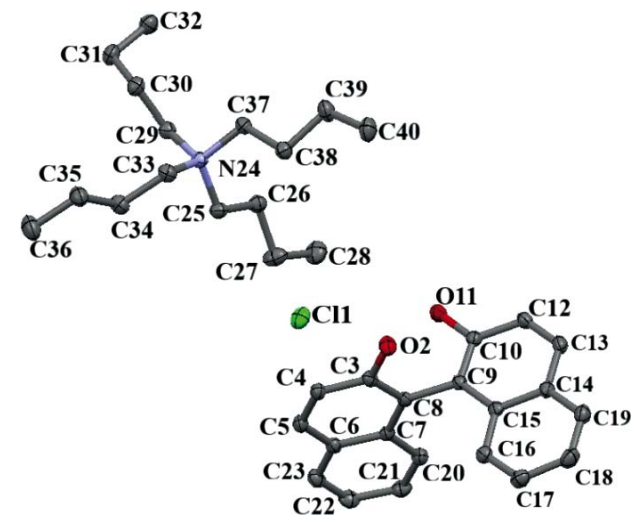

Fig. 2 Atom labeling scheme of 1:1 TBAC/BNP. H atoms are not shown for clarity purpose.

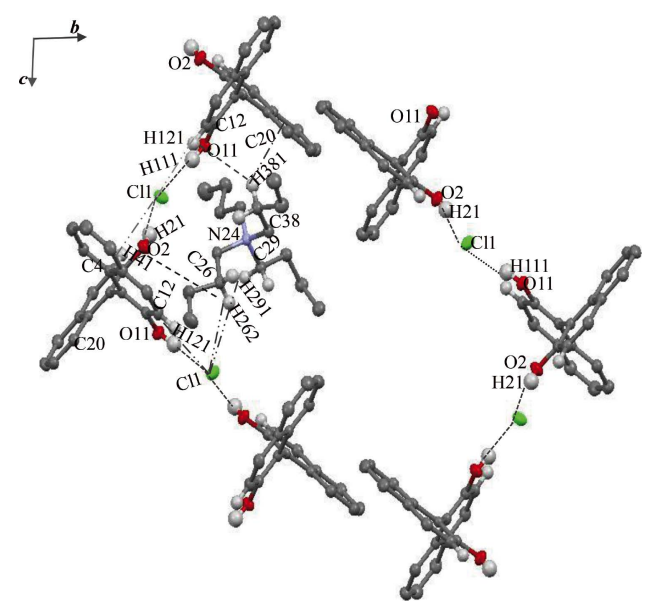

Fig. 3 Intermolecular interactions in the crystal structure of TBAC/ BNP. Some $\mathrm{H}$ atoms are omitted for clarity purpose. Crystal packing viewed along the $a$-axis.

(3.487(2) $\AA$ ) occurring between an H-atom of a methylene group with carbon in a phenyl moiety of BNP are also observed.

\section{Acknowledgements}

E. M.-O. would like to thank the Department of Chemistry, University of Oxford for support.

\section{References}

1. G. R. Desiraju, "Crystal Engineering: The Design of Organic Solids", 1989, Elsevier, Amsterdam.

2. E. Marfo-Owusu and A. L. Thompson, Mol. Crystallogr. Liq. Cryst., 2015, 607, 50.

3. K. Mori, Y. Matsuda, and S. Hashino, Acta Crystallogr, 1993, C49, 1224.

4. Q. Wang, A. Habenschuss, A. Xenopoulos, and B. Wonderlich., Mol. Cryst. Liq. Cryst. Sci. Technol. Sect. A, 1995, 264, 115.

5. F. Toda, K. Tanaka, T. Okada, S. A. Bourne, and L. R. Nassimbeni, Supramol. Chem., 1994, 3, 291.

6. A. K. Masood, A. W. McCulloch, and A. G. McInnes, Can. J. Chem., 1985, 63, 2119. 